\title{
Low-Speed Aerodynamic Characteristics of Supercritical Airfoil with Small High-Lift Devices from Flow Pattern Measurements
}

\author{
Thai Duong Nguyen, Masashi Kashitani, Masato Taguchi \\ Aerospace Department, National Defense Academy of Japan, Yokosuka, Japan \\ Email: em59046@nda.ac.jp
}

How to cite this paper: Nguyen, T.D., Kashitani, M. and Taguchi, M. (2020) Low-Speed Aerodynamic Characteristics of Supercritical Airfoil with Small High-Lift Devices from Flow Pattern Measurements. Journal of Flow Control, Measurement \& Visualization, 8, 159-172.

https://doi.org/10.4236/jfcmv.2020.84010

Received: March 27, 2020

Accepted: July 16, 2020

Published: September 23, 2020

Copyright () 2020 by author(s) and Scientific Research Publishing Inc. This work is licensed under the Creative Commons Attribution International License (CC BY 4.0).

http://creativecommons.org/licenses/by/4.0/

\begin{abstract}
In this paper, the lift coefficients of SC-0414 airfoil are estimated by applying modified Yamana's method to the flow visualization results, which are obtained by utilizing the smoke tunnel. The application of the modified Yamana's method is evaluated with two calculation methods. Additionally, the lift estimation, wake measurements, and numerical simulations are performed to clarify the low-speed aerodynamic characteristics of the SC airfoil with flaps. The angle of attack was varied from $-5^{\circ}$ to $8^{\circ}$. The flow velocity was $12 \mathrm{~m} / \mathrm{s}$ and the Reynolds number was $1.6 \times 10^{5}$. As a result, the estimated lift coefficients show a good agreement with the results from reference data and numerical simulations. In clean condition, the lift coefficients calculated from the two methods show quantitative agreement, and no significant difference could be confirmed. However, the slope of the lifts calculated from $y_{s}$ is higher and closer to the reference data than those obtained from $s c$, where $y_{s}$ denotes the height where the distance from the streamline to the reference line is the largest, and $s c$ denotes the displacement of the center of pressure from the origin of the coordinate, respectively. In the case of flaps, the GFs have an observable effect on the aerodynamic performance of the SC-0414 airfoil. When the height of the flap was increased, the lift and drag coefficients increased. The installation of a GF with a height equal to $1 \%$ of the chord length of the airfoil significantly improved the low-speed aerodynamic performance of SC airfoils.
\end{abstract}

\section{Keywords}

Supercritical Airfoil, Gurney Flap, Potential Flow, Flow Visualization

\section{Introduction}

Several experimental investigations on estimating the lift coefficient of airfoils in 
two-dimensional (2D) smoke tunnels have been performed [1]-[8]. The technique estimating the sectional lift coefficient from the flow visualization results in the 2D smoke tunnel was proposed by Yamana [1] [2], and it was successfully applied to the basic airfoil and the cases of flaps. In a smoke tunnel, the streamlines can be visualized as smoke lines. The lift coefficient can be estimated by measuring the distance from several points on a specific streamline to the reference line in the middle of the test section and by applying the measured values to the circulation equation. The origin of the coordinates is set at a quarter chord length from the leading edge of the airfoil. When a high-lift device, such as Gurney flap, is attached to an airfoil, the center of pressure displaces from the origin since effective camber varies due to the flap. In order to estimate the lift coefficient by Yamana's method, the displacement of center of pressure has to be taken into account. There are two calculation methods to evaluate it. The first one is that measures the displacement ( $s c$ ) of the center of pressure from the origin of the coordinate in the streamwise direction. Another is that considers the height $\left(y_{s}\right)$ where the distance from the streamline to the reference line is the largest. Yamana et al. [1] indicated that the lift coefficient estimated by the latter method is slightly smaller than the results of the first method.

In the original method, a smoke line must be adjusted to intersect with the reference line at a specific distance of upstream. It requires a long test section of smoke tunnel to visualize and adjust the intersection. Yamaguchi et al. [3] and Kashitani et al. [4] have developed a modified version of Yamana's method to estimate the lift coefficient of an airfoil in a condition of short test section that the intersection of the smoke line and reference line is not required. The modified method was carefully validated by conventional airfoil data. In the study, the method was applied to investigate the lift characteristics of a double-wedge airfoil and modified double-wedge airfoil [4]. To take into account the displacement of the center of pressure when a small high-lift device is attached to the airfoils, Kashitani et al. [5] [6] and Nguyen et al. [7] [8] calculated the lift coefficient by modified Yamana's method that considered the displacement (sc) of the center of pressure in the streamwise direction. The methods of obtaining the lift coefficient from $s c$ and from $y_{s}$ are both included in the original Yamana's method [1]. Although both methods are also applicable in the modified Yamana's method, the latter still has not been applied. In comparison with the measurement of the value $s c$, it is expected that $y_{s}$ can be measured within higher spatial resolution, because it basically tends to be larger measured value. It is considered that more accurate results can be obtained by the method using $y_{s}$.

Supercritical airfoils are widely used for civil transport aircraft. These airfoils first garnered attention when NASA made efforts to develop an airfoil that shows better performance in transonic flow while retaining acceptable characteristics in the low-speed flow [9]. Generally, supercritical airfoils in combination with flaps are considered to improve the aerodynamic performance of aircraft, especially during takeoff and landing [10]. The supercritical airfoils have asym- 
metric configuration and a camber near the trailing edge. With such configurations, the supercritical airfoil is considered as an appropriate target to examine the modified Yamana's method that considers the displacement of the center of pressure. In this study, the displacement of the center of pressure is controlled by varying angle of attack and by installing a small trailing-edge flap called Gurney flap (GF) aiming to investigate the scope of the applicability of the method. A Gurney flap (GF) is a simple and small flat plate installed at the trailing edge. The higher effective camber is obtained when the GF is installed. Studies about Gurney flaps have been performed and have demonstrated the impressive lift enhancement effect of the GF [11] [12] [13]. The results of these studies also showed that the use of a GF significantly increases the pre-stall lift and lift-to-drag ratio at small angles of attack. Understanding the aerodynamic characteristics of transonic airfoils with Gurney flaps at low-speed flow is also useful for aircraft designs.

In the present study, the flow visualizations around SC-0414 airfoil and estimation of lift coefficient are performed utilizing the low-speed smoke tunnel. The application of the modified Yamana's method is evaluated with two calculation methods based on the measurement of $s c$ and $y_{s}$. Furthermore, the lift estimation, wake measurements and numerical simulations are performed to clarify the low-speed aerodynamic characteristics of the SC airfoil with Gurney flaps.

\section{Modified Yamana's Method}

Figure 1 shows a detailed image of the modified Yamana's method [3]-[8]. The origin of the coordinates is set at a quarter chord length from the leading edge of the airfoil. In the figure, $c$ is the airfoil chord length, $h$ is the height of the test section, $y_{0}$ is the height of the smoke line at $x=-n c, y_{1}$ is the height of the smoke line, which closest to the airfoil, at $x=-c, s c$ is the horizontal displacement of the center of pressure, and $y_{s}$ is the height of the smoke line at $x=s c$. When a streamline passes through points $\mathrm{A}\left(-n \mathcal{c}, y_{0}\right), \mathrm{B}\left(-c, y_{1}\right)$, and $\mathrm{C}\left(\mathrm{sc}, y_{\mathrm{s}}\right)$, the following stream function is established:

$$
\psi\left[-(n+s) C, Y_{0}\right]=\psi\left[-(1+s) C, Y_{1}\right]=\psi\left[0, Y_{\mathrm{s}}\right]
$$

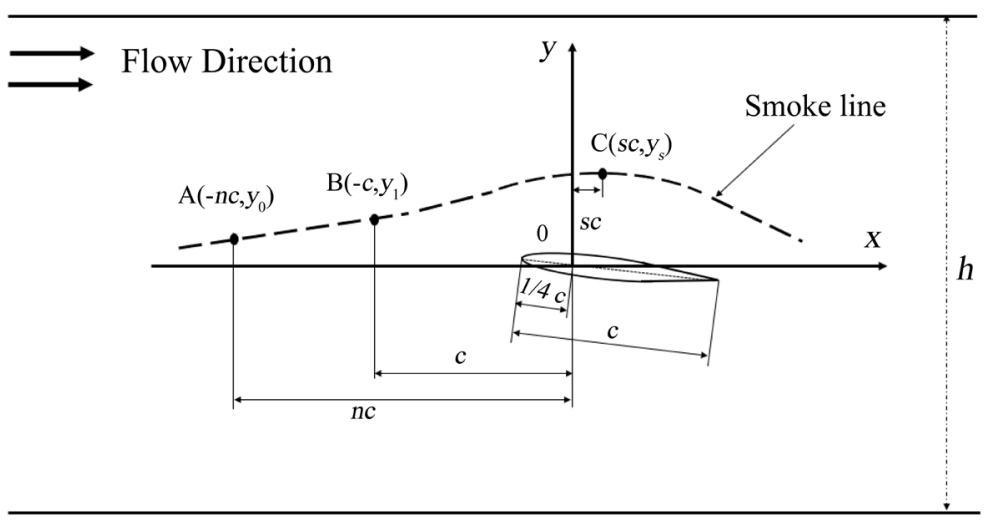

Figure 1. Schematic explanation of the modified Yamana's method. 
where $C=\pi c / h, Y_{0}=\pi y_{0} / h, Y_{1}=\pi y_{1} / h$, and $Y_{s}=\pi y_{s} / h$. Then, with the inclusion of the flap effects, the lift coefficient can be calculated as:

$$
c_{l}\left(Y_{0}, Y_{1}, s C\right)=4 \pi \frac{\left(Y_{1} / C-Y_{0} / C\right)}{G\left(Y_{0}, Y_{1}, s C\right)}
$$

where:

$$
G\left(Y_{0}, Y_{1}, s C\right)=\ln \frac{\left(\sinh ^{2}(n+s) C+\sin ^{2} Y_{0}\right)^{\frac{1}{2}}}{\left(\cosh (n+s) C+\cos Y_{0}\right)}-\ln \frac{\left(\sinh ^{2}(1+s) C+\sin ^{2} Y_{1}\right)^{\frac{1}{2}}}{\left(\cosh (1+s) C+\cos Y_{1}\right)}
$$

The lift coefficient can thus be calculated from the measured values of $y_{0}, y_{1}$, and $s c$.

Next, through the application of the modified Yamana's method, the calculation of the lift coefficient from $y_{s}$ is considered. With the same variable definitions as above, we have:

$$
\begin{aligned}
& c_{l}\left(Y_{0}, Y_{\mathrm{s}}, s C\right)=4 \pi \frac{\left(Y_{\mathrm{s}} / C-Y_{0} / C\right)}{G\left(Y_{0}, Y_{\mathrm{s}}, s C\right)} \\
& c_{l}\left(Y_{1}, Y_{\mathrm{s}}, s C\right)=4 \pi \frac{\left(Y_{\mathrm{s}} / C-Y_{1} / C\right)}{G\left(Y_{1}, Y_{\mathrm{s}}, s C\right)}
\end{aligned}
$$

With Equations (4) and (5), with sc and $c_{l}$ as the variables, the lift coefficient $c_{l}$ can be calculated from the measured values of $y_{0}, y_{1}$, and $y_{s}$. The lift coefficient can thus be calculated from the measured values of $y_{0}, y_{1}$, and $s c$.

In this study, we focus on investigating the modified Yamana's method by considering $y_{s}$, which intended to estimate the lift coefficient with higher accuracy.

In this investigation, the value of $n$ is 1.7 , and the airfoil chord length $c$ is 200 $\mathrm{mm} . n c$ is a distance from the origin of the coordinates to point $\mathrm{A}$, which is free to be determined in the front of point $\mathrm{C}$, as described in Figure 1. To easily measure the height $y_{0}$ of point $A$, we put $n$ as a value of 1.7. In the Yamana's experiments [1] [2], $n c$ is a distance where the point A must be adjusted to $y_{0}=0$ and the value of $n$ is about 4.5. Besides, $s$ is determined as the ratio between the airfoil chord length and the distance from the origin to point $\mathrm{C}$, where the height of the smoke line is largest. The value of $s$ changes when the measurement condition changes.

The lift coefficients estimated by the Yamana's method showed a good agreement with other experimental results when the smoke lines flow smoothly around the upper surface of the airfoil [1]-[6]. When flow separation occurs, the unsteady characteristic becomes large. However, the calculation result also shows a qualitatively similar trend with the other results [4] [6]. Also, further considerations are required to investigate the effects of high velocity on the accuracy of the method. However, it seems that there is no upper-speed limit for using this method in theory. 


\section{Experimental setup}

\subsection{Smoke wind Tunnel and Airfoil Model}

Figure 2(a) and Figure 2(b) show the smoke wind tunnel used in this experiment. The tunnel is a suction-type tunnel. The width, height, and length of the test section are 150, 1500, and $2000 \mathrm{~mm}$, respectively. The maximum wind velocity is $26 \mathrm{~m} / \mathrm{s}$. The airfoil was fixed on a turntable to allow the angle of attack to be easily changed. Smoke was generated by a smoke generator (SC-3, Kanto Kagaku). A Nikon D50 camera with a shutter speed of $1 / 15 \mathrm{~s}$ was used to record the flow pattern. The wind tunnel has 51 smoke lines with $20 \mathrm{~mm}$ of the interval.

Figure 3(a) shows the configuration of the SC-0414 airfoil model. The chord length of the airfoil model is $200 \mathrm{~mm}$, and the wingspan is $150 \mathrm{~mm}$, which is the same as the width of the wind tunnel. Figure 3(b) shows the SC-0414 airfoil with Gurney flap, which used in this experiment. The heights of the flaps $I$ were 2,4 , and $6 \mathrm{~mm}$, which correspond to $1 \%, 2 \%$, and $3 \%$ of the airfoil's chord length, respectively. The thickness of the flaps is $0.05 \mathrm{~mm}$. The Gurney flaps were attached to the trailing edge of the airfoils with scotch tape.

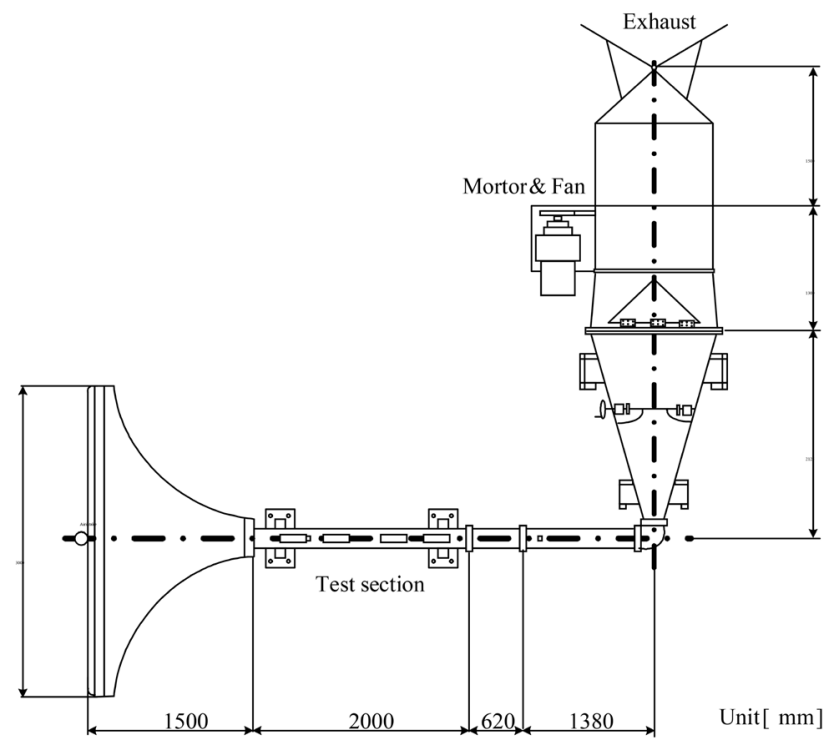

(a) Top view.

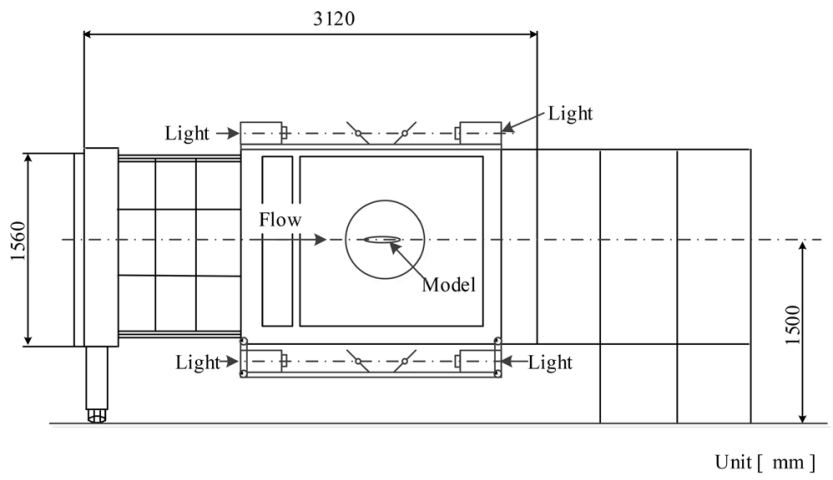

(b) Side view.

Figure 2. 2D smoke wind tunnel. 


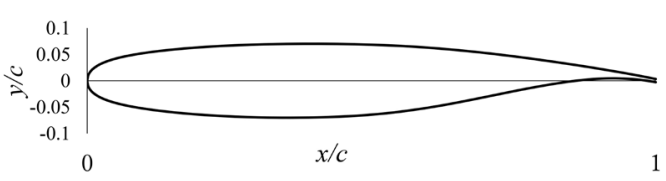

(a) SC-0414 airfoil

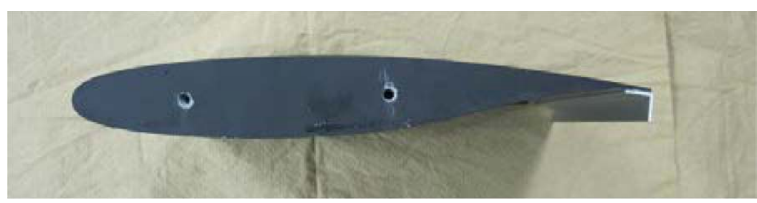

(b) SC-0414 airfoil with Gurney Flap

Figure 3. Experimental models.

\subsection{Wake Measurement}

The drag can be obtained by comparing the momentum in the air upstream of the model with that downstream of the model [14]. The drag coefficient $c_{d}$ is then given by

$$
c_{d}=\frac{1}{c} \int_{a}^{b}\left(\frac{u_{2}}{u_{1}}-\frac{u_{2}^{2}}{u_{1}^{2}}\right) \mathrm{d} y
$$

where $u_{1}$ is the inflow velocity, $u_{2}$ is the outflow velocity, $[a, b]$ represents the measurement range in the wake of the test model, $c$ is the chord length, and $y$ is the position in the vertical direction.

A hot-wire system was used to measure the outflow velocity in the wake of the airfoil. A hot-wire anemometer (System 7000, Kanomax) was used in this study, and a I-type hot film (0251R-T5) was selected to measure the velocity. The data measured by the hot-wire system were transmitted to a computer by a data logger (NR500, Keyence).

\subsection{Experimental Condition}

The experimental condition is shown in Table 1. Flow visualizations were performed at angles of attack of $-5^{\circ}$ to $8^{\circ}$. The tunnel flow velocity $U_{\infty}$ is $12 \mathrm{~m} / \mathrm{s}$, and the Reynolds number Re based on the chord length was $1.6 \times 10^{5}$. In wake measurement, the probe was set up $1.0 c$ downstream from the trailing edge of the airfoil model. The probe was swept in the vertical direction over a measurement range $[a, b]$ of $300 \mathrm{~mm}(-150 \mathrm{~mm} \leq y \leq 150 \mathrm{~mm}$; where $y=0$ is a point located in the reference line in Figure 1) at $5 \mathrm{~mm}$ intervals. The outflow velocity was measured for $20 \mathrm{~s}$, and the average over the measurement period was used to calculate the drag coefficient. The data collection frequency is $10 \mathrm{~Hz}$. The numerical simulation setup is the same as the conditions in the wind tunnel experiment.

\section{Numerical Simulation}

Numerical simulations are performed to compare with experimental results. Figure 4 shows the grid distribution of the simulation. The chord length of the 


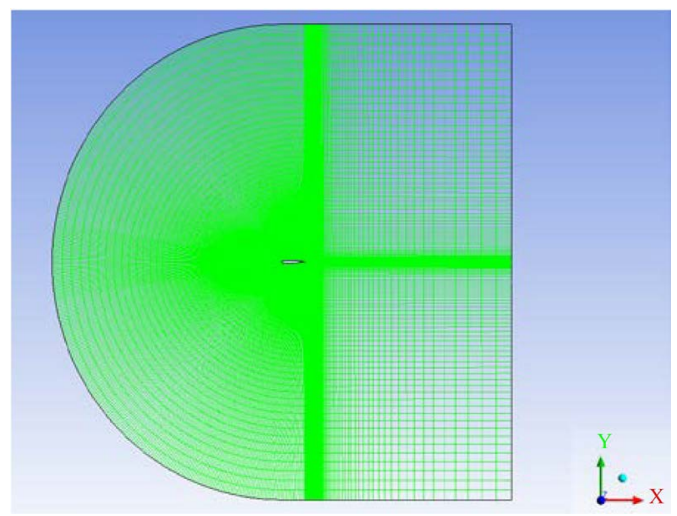

Figure 4. C-type grid distribution around the airfoil.

Table 1. Experimental condition.

\begin{tabular}{ccc}
\hline & \multicolumn{2}{c}{ Experimental condition } \\
\hline & Flow velocity & $12 \mathrm{~m} / \mathrm{s}$ \\
\hline \multirow{2}{*}{$\begin{array}{c}\text { Angle of } \\
\text { attack }\end{array}$} & Flow visualization & SC-0414 with GFs \\
& Wake measurement & $-5^{\circ}-8^{\circ}\left(1^{\circ}\right.$ interval $)$ \\
Wake & Wake location & $-4^{\circ}-8^{\circ}\left(2^{\circ}\right.$ interval $)$ \\
measurement & Measurement range & $1.0 c$ from the trailing edge of the airfoil model \\
& Measurement interval & $-150 \mathrm{~mm} \leq y \leq 150 \mathrm{~mm}$ \\
& Measurement time at 1 point & $\Delta y=5 \mathrm{~mm}$ \\
\end{tabular}

SC-0414 airfoil model is $200 \mathrm{~mm}$. ICEM software was used to construct a C-type structured grid with 340 and 400 points distributed on the surface of the airfoil alone and the airfoil with the GF, respectively.

Upper and lower boundaries of the simulation domain are 10 chord-length away the airfoil, as the velocity inlet condition and downstream outflow boundary. The $y+$ value of the grid is approximately 1 , which means that the height of first grid element nearest the airfoil is approximately $2.4 \times 10^{-5} \mathrm{~m}$. Simulations were performed using the software Ansys Fluent 18.2. The Reynolds-averaged Navier-Stokes (RANS) equation and the $k$ - $\omega$ shear stress transport (SST) model was applied in all conditions [15]. The pressure-implicit with splitting of operators (PISO) algorithm was used to steady the simulation. The second-order pressure scheme, third-order upwind scheme for conservation laws (MUSCL), and first-order viscosity scheme were selected [11]. The convergence criteria were selected as $10^{-6}$ for the continuity and $10^{-5}$ for other terms.

\section{Results and Discussion}

\subsection{Flow Visualization}

Figure 5(a) shows the flow fields around the SC-0414 airfoil models with installed GFs at an angle of attack $\alpha$ of $5^{\circ}$. Figure 5(a-1) shows the case with no flap, and Figures 5(a-2)-(a-4) show the cases with $1 / c=0.01,0.02$, and 0.03 , re- 
spectively, where $l$ is the height of the Gurney flap and $c$ is the chord length of the airfoil model. The horizontal yellow lines in the photographs are reference lines, and the contrast of the photographs has been adjusted. The smoke lines flow smoothly on the upper surface of the airfoil. The curves of the smoke lines approaching the leading edge of the airfoil changed as the height of the GF were increased. As shown in Figure 5(a-1), the smoke lines were totally separated from the reference line in front of the airfoil when no flap was present. In Figure $5(\mathrm{a}-2)$, the smoke lines can be seen intersecting the reference line as a result of the flap. As shown in Figure 5(a-3) and Figure 5(a-4), with the larger GFs, the distance between the smoke lines and the airfoil were larger than with the smaller flap in Figure 5(a-2), and the smoke lines flowed smoothly around the airfoil.

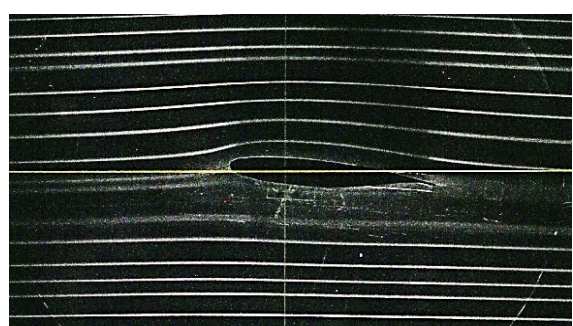

(a-1) SC-0414 airfoil with no flap



(a-2) SC-0414 airfoil with $/ / c=0.01$

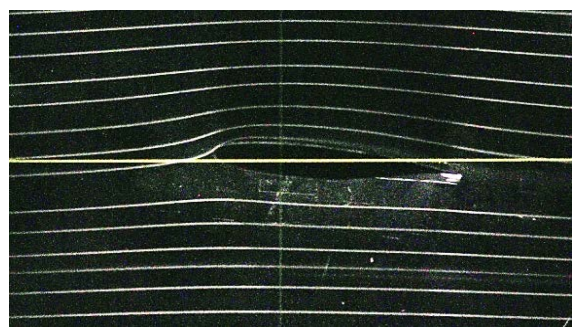

(a-3) SC-0414 airfoil with $/ / c=0.02$

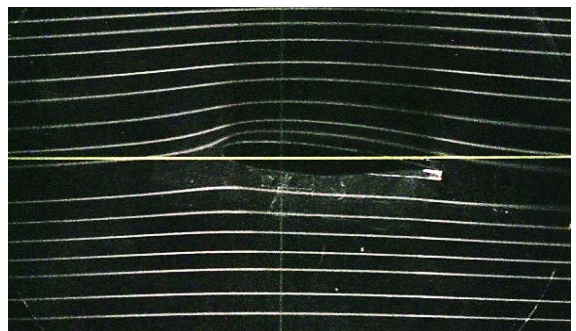

(a-4) SC-0414 airfoil with $/ / c=0.03$

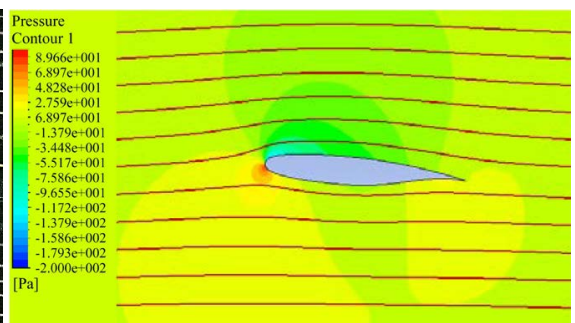

(b-1) SC-0414 airfoil with no flap

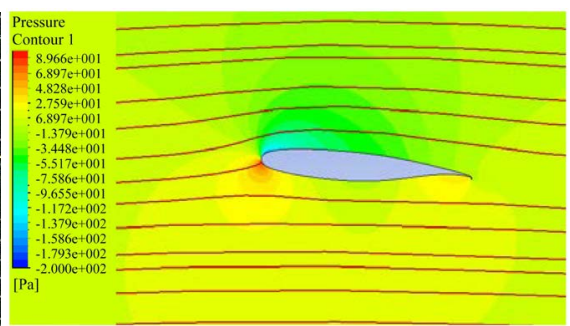

(b-2) SC-0414 airfoil with $/ / c=0.01$



(b-3) SC-0414 airfoil with $/ / c=0.02$

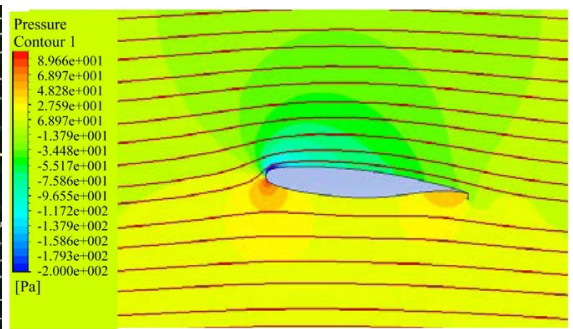

(b-4) SC-0414 airfoil with $/ / c=0.03$

Figure 5. (a) Flow visualization around airfoil models $\left(\alpha=5^{\circ}\right)$. (b) Pressure contours and streamlines obtained by numerical simulation $\left(\alpha=5^{\circ}, U_{\infty}=12 \mathrm{~m} / \mathrm{s}, R e=1.6 \times 10^{5}\right)$. 
When the smooth smoke lines can be observed, the estimated lift coefficients showed a good agreement with other experimental results [1]-[6]. The effects of flaps change the appearances of the line of the smoke, and the estimated lift will be different.

Figure 5(b) shows the pressure contours and streamlines calculated by Ansys Fluent. The red lines represent the streamlines. As in Figure 5(a), the angle of attack $\alpha$ is $5^{\circ}$. The flap heights in the images are also the same as in Figure 5(a). Because of the original camber of the SC airfoil, a high-pressure region at the lower surface of trailing edge can be observed in Figure 5(b). Figure 5(b) shows that the pressure at the upper and lower surfaces varies with the GF height. In Figure 5(b-4), a large high-pressure area is observable at the lower surface, and the structure of the low-pressure area on the upper surface is different from those in Figure 5(b-1)-(b-3). A comparison of Figure 5(a) and Figure 5(b) shows that there is qualitative agreement between the visualization results of the smoke wind tunnel experiment and the numerical simulation results. Also, in Figure 5(b), when increasing the height of the flaps, the area of high pressure is expanded at leading and trailing edge due to the effects of increasing the camber.

\subsection{Estimation of Lift Coefficient}

Figure 6(a) shows the lift coefficient $c_{l}$ at different angles of attack $\alpha$ in the case of no flap. In previous study of the modified Yamana's method in this experimental system [4], the validation is conducted in the case of NACA 0012 airfoil. The results show a good agreement with other experimental results. In Figure $6(\mathrm{a})$, the results are plotted together with reference data predicted using XFOIL software at a Reynolds number Re of $2 \times 10^{5}$ (airfoil tools [16]) for the sake of comparison. In the experiments, the measurements were made after the pattern of the smoke lines around the airfoil model became nearly steady. To confirm that, three pictures were taken at every angle of attack. The values of specific points in the three pictures are separately measured. The height $y_{0}, y_{1}, y_{s}$ were determined as their average values. The variation of these values in the three pictures was not significant. Then, the average values are applied to the equation (2), (3), (4), (5) in section 2. The slopes of the lift obtained from the reference data and numerical simulation with respect to the angle of attack are approximately 0.118 and 0.103 , respectively. Additionally, the slopes for the experimental results obtained from $s c$ and $y_{s}$ are 0.113 and 0.117 , respectively. This demonstrates that the experimental results show a good agreement with the numerical simulation and reference XFOIL data results. The simulation results quantitatively agreed with the reference data for angles of attack from $0^{\circ}$ to $8^{\circ}$. However, the XFOIL lift coefficients are less than those obtained from the numerical simulation at negative angles of attack. In contrast, the lift coefficients calculated from $s c$ and $y_{s}$ show quantitative agreement, and no significant difference between these results could be confirmed. However, the slope of the lift calculated from $y_{s}$ is higher and closer to the reference data than those obtained 


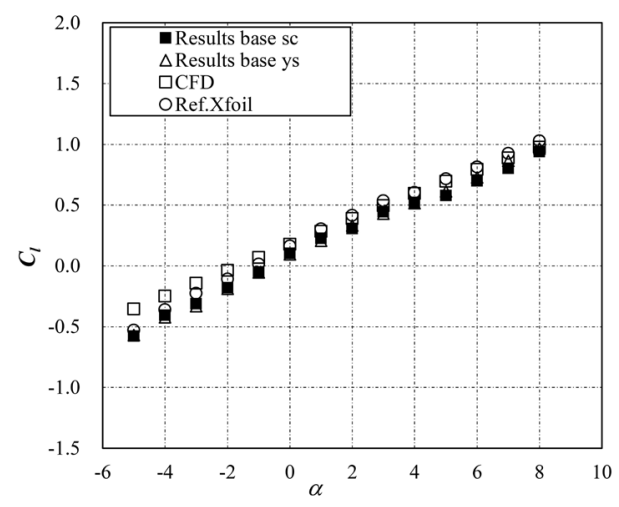

(a) Experimental and analytical lift coefficient results.

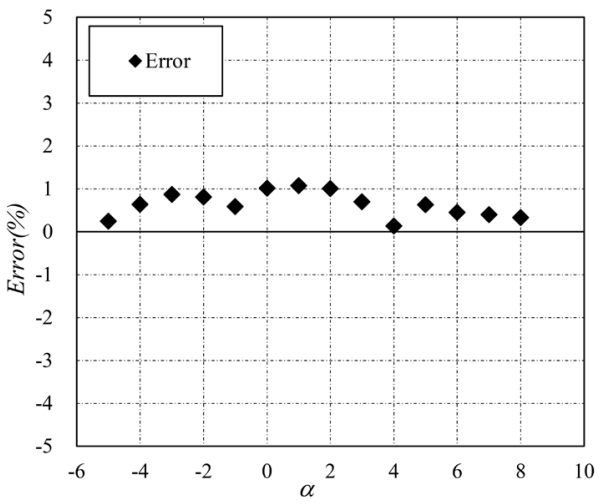

(b) Error of the center of pressure displacement.

Figure 6. Modified Yamana's method on estimating lift coefficient of SC-0414 airfoil.

from $s c$. Since the distance of $y_{s}$ is larger than $s c$, it is considered that the measurement of $y_{s}$ can obtain a better resolution, and the measurement of $y_{s}$ is easier than $\mathrm{s} c$.

Figure 6(b) shows the error of the displacement of the center of pressure in the SC-0414 airfoil experiment. The errors were calculated as

$$
\text { Error }=\frac{\left|\mathrm{s} c_{\text {cal }}-\mathrm{s} c_{\text {mea }}\right|}{c}
$$

where $s c_{c a l}$ is the displacement of the center of pressure obtained from the Equations (4) and (5). The value $y_{s}$ can be directly measured by G3 data software from the pictures. Then, Equations (4) and (5) becomes a set of 2 equations with 2 variables $\left(c_{l}\right.$ and $\left.s c\right)$. The set of the two equations that can be solved by using the Matlab program. The value of "sc calculated by the Matlab program is called $s c_{c a l}$. The value of " $s c$ " directly measured from point $\mathrm{C}$ to the origin of the coordinates is called $s c_{\text {mea }}$. This value is applied to equation (2) to calculate the lift coefficients, which are called the "results base $s c$ ". Overall, the maximum error was within approximately $1 \%$ regardless of the angle of attack, demonstrating the good agreement between the calculation and measurement results. The results also show the accuracy of the modified Yamana's method considering the displacement of center pressure in the case of the SC-0414 airfoil. 


\subsection{Aerodynamic Characteristics of SC Airfoil with GF}

Figure 7(a) shows the lift coefficient results obtained by the flow pattern technique and numerical simulation. The calculation method based on $s c$ in the case of flaps was clarified in the previous studies [5] [6]. In this study, the lift coefficient results were obtained using the modified Yamana's method based on the height $y_{s}$, as were the airfoil baseline's results in Figure 6(a). The lift coefficients were calculated from the measured vertical distance $y_{s}$ of the smoke line averaged over three measurements.

The slope of the lift coefficient from the simulation results is approximately 0.118 and is nearly the same for all flap height conditions. Because the modified Yamana's method is effective in steady flow, this investigation was conducted at angles of attack ranging from $-5^{\circ}$ to $8^{\circ}$. The case of the airfoil with $l / c=0.01$ shows that the addition of a flap significantly improves the lift. From $4^{\circ}$ to $8^{\circ}$, in all cases, the experimental results were slightly different from the corresponding simulation results; however, the results showed overall good qualitative agreement. The experimental results showed the same trend as the simulations. An increase in the height of the flap produced an increase in the lift coefficient. The results show that in the case of the SC-0414 airfoil, the GF has similar effects as other simple plain flaps, as has been discussed by Raymer [17].

Figure 7(b) shows the drag coefficient results obtained from the wake measurement experiment and the numerical simulation. The experimental results were calculated using the wake measurement method explained above. The lowest drag coefficients among the four GF cases were obtained in the case of the airfoil without a GF. As the height of the flap was increased, the drag coefficient increased. However, because the presence of a flap causes a change in the camber line of the airfoil, the angles of attack corresponding to the minimum drag in each case are different, as shown clearly in the simulation results. The angle of attack corresponding to the minimum drag was $0^{\circ}$ in case of no flap, $-2^{\circ}$ in the case with $l / c=0.01$, and approximately $-4^{\circ}$ in the case with $l / c=0.02$ and 0.03 . In case with $l / c=0.03$, the experimental results were slightly different from the simulation results. However, overall, the experimental results showed the same trends as the simulation results.

Figure $7(\mathrm{c})$ shows the lift-to-drag ratio results. These results were calculated from the lift and drag coefficients shown in Figure 7(a) and Figure 7(b). Increasing the height of the GF causes both the drag and the lift to increase. In comparison with the increase in the lift, the increase in the drag is relatively small. Therefore, when the height of the flap was increased, the lift-to-drag ratio increased with increasing angle of attack from $-4^{\circ}$ to $8^{\circ}$. The lift-to-drag ratios were maximized at an angle of attack of approximately $6^{\circ}$. In the simulation results, from $2^{\circ}$ to $8^{\circ}$, the lift-to-drag ratio results were nearly the same for all the three considered flap heights. However, the lift-to-drag ratio in the case with $1 / c$ $=0.01$ was slightly larger than those in the other cases, indicating a significant improvement in the lift-to-drag ratio relative to the case with no flap. The experimental results show a similar trend as the simulations. 




(a) Lift coefficient.



(b) Drag coefficient.

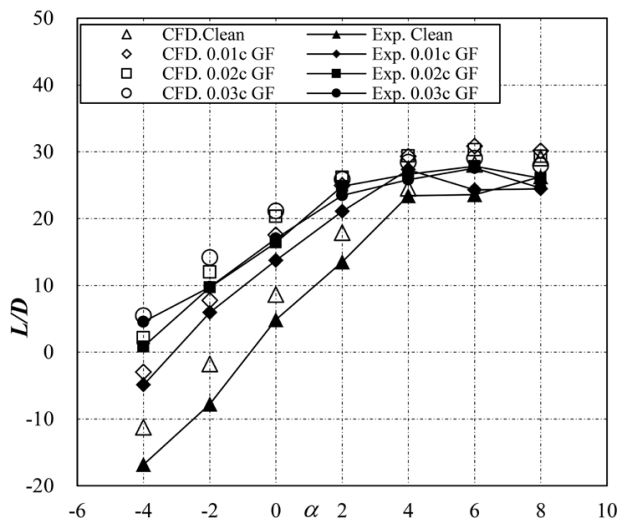

(c) Lift-to-drag ratio.

Figure 7. Effects of the GF height on aerodynamic performance.

\section{Conclusions}

In this study, the flow visualization around SC-0414 airfoil and estimation of lift coefficient are performed utilizing the low-speed smoke tunnel. The application of the modified Yamana's method is validated with two calculation methods. Additionally, the lift estimation, wake measurements and numerical simulations are performed to clarify the low-speed aerodynamic characteristics of the SC 
airfoil with Gurney flaps. The results are as follows:

1) The visualization results from the smoke wind tunnel experiment and the numerical simulation results showed a qualitative agreement. When increasing the height of the flaps, the area of high pressure is expanded at the leading and trailing edge due to the effect of increasing the camber.

2) The lift coefficient calculated by modified Yamana's method shows good agreement with the numerical simulation results and reference data. The methods of obtaining the lift coefficient from the height $y_{s}$ where the distance from the streamline to the reference line is the largest can obtain closer results to reference data than the method based on the displacement $s c$ of the center of pressure.

3) In modified Yamana's method calculation, the maximum error of the value sc was approximately $1 \%$ regardless of the angle of attack, demonstrating the agreement between the calculation and measurement results.

4) When the height of the flap was increased, the lift and drag coefficients increased. The installation of a GF with a height equal to $1 \%$ of the chord length of the airfoil significantly improved the low-speed aerodynamic performance of SC airfoils.

\section{Conflicts of Interest}

The authors declare no conflicts of interest regarding the publication of this paper.

\section{References}

[1] Yamana, M. (1965) Measurement of Section Lift Coefficient in a Two-Dimensional Smoke Wind-Tunnel. Journal of the Japan Society for Aeronautical and Space Sciences, 13-141, 321-325.

[2] Yamana, M. and Nakaguchi, H. (1979) Theory of Airplane Design. Yokendo Press, Tokyo, 645-648. (In Japanese)

[3] Yamaguchi, Y., Kashitani, M. and Sato, K. (2002) An Experiment on Aerodynamic Characteristics of Airfoil Constructed with Flat Plates in a Low Reynolds Number Flow. Bulletin of National Defense Academy, 40-9, 117-126.

[4] Kashitani, M. and Yamaguchi, Y. (2003) Visualization around Double Wedge Airfoil Models in a Two Dimensional Smoke Wind Tunnel. Journal of Visualization Society of Japan, 23, 100-106. (In Japanese) https://doi.org/10.3154/tvsj.23.100

[5] Kashitani, M., Yamaguchi, Y., Kai, Y., Hirata, K. and Kusunose, K. (2008) Preliminary Study on Lift Coefficient of Biplane Airfoil in Smoke Wind Tunnel. AIAA Paper 2008-349. https://doi.org/10.2514/6.2008-349

[6] Kashitani, M., Yamaguchi, Y., Kai, K. and Kusunose, K. (2010) Study on Busemann Biplane Airfoil in Low Speed Smoke Wind Tunnel. Transactions of the Japan Society for Aeronautical and Space Sciences, 52, 213-219. https://doi.org/10.2322/tjsass.52.213

[7] Nguyen, T.D., Kashitani, M. and Taguchi, M. (2019) Aerodynamic Investigations on SC-0414 Airfoil with Small High Lift Devices. Journal of Physics. Conference Series, 1215, Article ID: 012004. https://doi.org/10.1088/1742-6596/1215/1/012004

[8] Nguyen, T.D., Kashitani, M. and Taguchi, M. (2018) Preliminary Investigation on 
Estimating Lift of SC-0414 Airfoil by Utilizing Smoke Wind Tunnel. The 56th Aircraft Symposium, Yamagata, 14-16 November 2018, 3A10. (In Japanese)

[9] Harris, C.D. (1990) NASA Supercritical Airfoils, a Matrix of Family-Related Airfoils. NASA Technical Paper 2969.

[10] Melton, L., Schaeffler, N.W. and Lin, J.C. (2007) High-Lift System for a Supercritical Airfoil: Simplified by Active Flow Control. AIAA Paper 2007-707.

[11] Yu, T., Wang, J.J. and Zhang, P.F. (2011) Numerical Simulation of Gurney Flap on RAE-2822 Supercritical Airfoil. Journal of Aircraft, 48, 1565-1575. https://doi.org/10.2514/1.C031285

[12] Maughmer, M.D. and Gotz, B. (2008) Experimental Investigation of Gurney Flaps. Journal of Aircraft, 45, 2062-2067. https://doi.org/10.2514/1.37050

[13] Daniel, L. and Traub, L.W. (2013) Effect of Aspect Ratio on Gurney-Flap Performance. Journal of Aircraft, 50, 1217-1225. https://doi.org/10.2514/1.C032140

[14] Anderson Jr., J.D. (2011) Fundamentals of Aerodynamics. Fifth Edition in SI Units, Chapter 2, 135-141.

[15] Menter, F.R. (1994) Two-Equation Eddy-Viscosity Turbulence Models for Engineering Applications. AIAA Journal, 32, 1598-1605. https://doi.org/10.2514/3.12149

[16] http://airfoiltools.com/airfoil/details?airfoil=sc20414-il\#polars

[17] Raymer, D.P. (2012) Aircraft Design: A Conceptual Approach. Fifth Edition, AIAA Educational Series, 419-428. https://doi.org/10.2514/4.869112

\section{Nomenclature}

$c=$ the airfoil chord length

$c_{d}=$ drag coefficient

$c_{l}=$ lift coefficient

$h=$ the height of the test section

$y_{0}=$ the height of the smoke line at $x=-n c$

$y_{1}=$ the height of the smoke line at $x=-c$

$R e=$ Reynolds number based on chord length

$s c=$ the horizontal displacement of the center of pressure

$u_{1}=$ the inflow velocity $\left(=U_{\infty}\right)$

$u_{2}=$ the outflow velocity

$x, y=$ Cartesian coordinated system

$\alpha=$ angle of attack

$y_{s}=$ the height of the smoke line at $x=s c$

$\psi=$ Stream function 